\title{
Solvent-free Cyanosilylation of Carbonyl Compounds Catalyzed by $\mathrm{NbCl}_{5}$
}

\author{
Soney C. George and Sung Soo Kim*

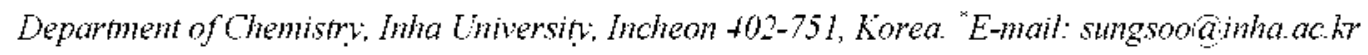 \\ Received March 16, 2007
}

\begin{abstract}
A simple and convenient method for the addition of TMSCN to carbonyl compounds is described. $\mathrm{NbCl}_{s}$ is found to possess a strong Lewis acid property to transform carbonyl compounds smoothly to the corresponding cyanosilylether in high yields (up to $99 \%$ ) in solvent- free conditions.
\end{abstract}

Key Words : Cyanohydrins. Aldehydes. Ketones. Solvent-free. $\mathrm{NbCl}_{s}$

\section{Introduction}

Cyanosilylation of carbonyl compounds is one of the most efficient methods for the synthesis of silyated cyanohydrins. It is well known that cyanohydrins are important intermediates for the synthesis of $\alpha$-hydroxy aldehydes. $\alpha$-hydroxyacids $\beta$-aminoalcohlos $\alpha$-cyanoketones. etc. ${ }^{.}$Several reagents including Lewis acids. Lewis bases. metal alkoxides. bifunctional catalysts. iodine. inorganic salts have been found to effectively transfer the cyano group from TMSCN to carbonyl compounds. " But in many of the reported results presence of solvent and additives are essential for the cyanosilylation reactions. ${ }^{3}$ In this connection we have developed several chiral ${ }^{4}$ and achiral ${ }^{5}$ catalysts for cyanosily lation of carbonyl compounds. There are various metal halides such

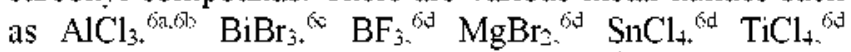

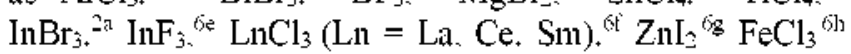
and $\mathrm{LiCl}^{\text {si }}$ which are acting as Lewis acid catalysts for cyanosily lation of aldehydes. Recently $\mathrm{NbCl}_{\Sigma}$ has emerged as an efficient Lewis acid catalyst in promoting various organic transformations. ${ }^{7}$ However no report has described so far the catalytic properties of $\mathrm{NbCl}_{3}$ for cyanosilylation reactions. We wish to herein report a simple and efficient method for the synthesis of silylcyanoethers in presence of cataly tic amount of $\mathrm{NbCl}_{\Sigma}$ at rt in solvent- free conditions.

\section{Results and Discussion}

The catalytic activity of $\mathrm{NbCl}_{5}$ has been tested for the reaction of benzaldehyde and TMSCN at it. As shown in Table 1. $\mathrm{NbCl}_{\Sigma}$ exhibits excellent catalytic activity under solvent- free conditions. $0.5 \mathrm{~mol} \%$ of $\mathrm{NbCl}_{\text {s }}$ gives sily lether of $97 \%$ in 30 minutes (entry 2). With $1 \mathrm{~mol} \%$ catalyst the yield was increased to $99 \%$ with duration of 15 minutes. The reaction time could be reduced from $15 \mathrm{~min}$ to $7 \mathrm{~min}$ by increasing the catalyst amount from $1 \mathrm{~mol} \%$ to $3 \mathrm{~mol} \%$. In presence of $\mathrm{CH}_{2} \mathrm{Cl}_{2}$ as solvent the reaction took 40 minutes to complete and yield was reduced to $94 \%$. Consequently 1 $\mathrm{mol} \%$ catalytic loading is considered optimal for present reactions. A series of carbonyl compounds react with TMSCN in the presence of $\mathrm{NbCl}_{\Sigma}$ under solvent-free condi-

$\overline{{ }^{a} \text { Visiting Scientisit from Department of Basic Science, Amal Jyothi }}$ College of Engineering. Koovapally, Kerala. India
Table 1. Cyanosily lation of benzaldehyde under various conditions ${ }^{s}$

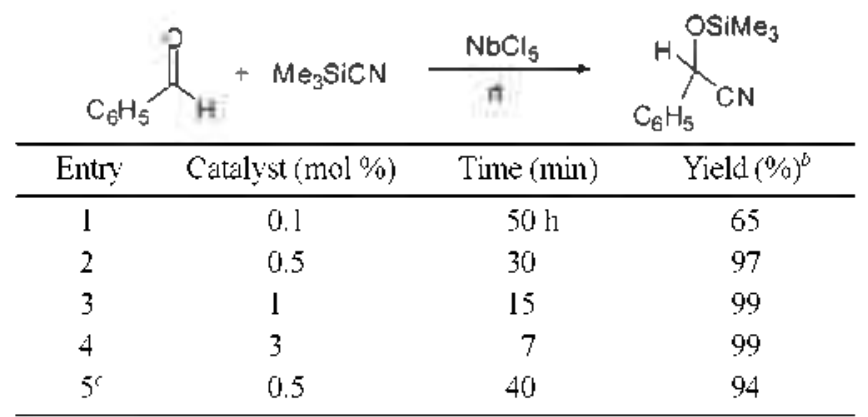

NbCls is added to a misture of 1 mmole of the benzaldehyde and 1.2 equiv. TMSCN. hisolated yield. ' in presence of $\mathrm{CH}_{2} \mathrm{Cl}_{2}$

tions at $\mathrm{rt}$ to give the cyanation products by utilizing the condition of entry 3 of Table 1.

Most of the aromatic and aliphatic aldehydes are converted into the corresponding cyanohydrin trimethylsilylether for relatively short reaction time in good to excellent isolated yields (entries 1-14) at rt. $p$-Tolualdehyde took less reaction time than $m$-tolualdehyde with $94 \%$ yield (entry 2 . 3). The reaction of $p$-methoxy benzaldehyde completed in 40 min with $65 \%$ yield. On the other hand the reaction of $p$ tert-butyl benzaldehyde took 120 minutes to complete the reaction with yield of $85 \%$. We have also noticed the influence $u$-. $p$ - and $m$-chlorosubstituted benzaldehyde on cyanosilylation reaction (entry $6,7,8$ ). o-Chloro benzaldehyde completed the reaction with 30 minutes with yield of $70 \%$ whereas the $p$ - and $m$-chloro benzaldelydes completed the reaction within 10 minutes but the yield is reduced to $65 \%$ and $58 \%$ respectively. $m$-Phenosybenzaldehyde took 15 minutes to obtain $83 \%$ yield [entry 9]. Acid sensitive 2fulfuraldehy'de [entry 10 ] gives the product with $65 \%$ yield at $40 \mathrm{~min}$. This may indicate that the catalytic system selectively activate the carbonyl function and keep the furan ring intact. The reaction of naphthaldehyde with TMSCN was completed within 40 min with yield of $92 \%$ (entry 11 ). The silylcyanation of isobutryaldehyde was completed within 60 minutes with TMSCN with yield of $84 \%$. Crotonaldehy'de and cinnamaldehyde [entry $13 \& 14$ ] were predominately converted into 1,2 adducts leaving the olefinic function intact. No conjugated addition product was observed. Unsubstituted benzaldehyde takes $15 \mathrm{~min}$ (entry 1) for the cyanosilylation which is the best result of present 
Table 2. Cranosilylation of various aldelydes and ketones with $\mathrm{NbCl}^{2}$

Solvent -free, $\mathrm{rt}$ Tine (min)

"I mmole of the benzaldelyyde. $1 \mathrm{~mol}$ ". of $\mathrm{NbCl}_{5} .1 .2$ equir. TMSCN are stirted together. "isolated vield, ( $100^{\circ}, 0$ conversion according to ${ }^{1} \mathrm{H}$ NMR analysis)

reactions in terms of reaction time and yield.

We have also examined the catalytic activity of $\mathrm{NbCl}_{5}$ for several ketones (entries 15.16\& 17). The 2-cyclohexen-1one completed the reaction within 15 minutes but the yield is $83 \%$ (entry 15). 2-Octanone completed the reaction with 380

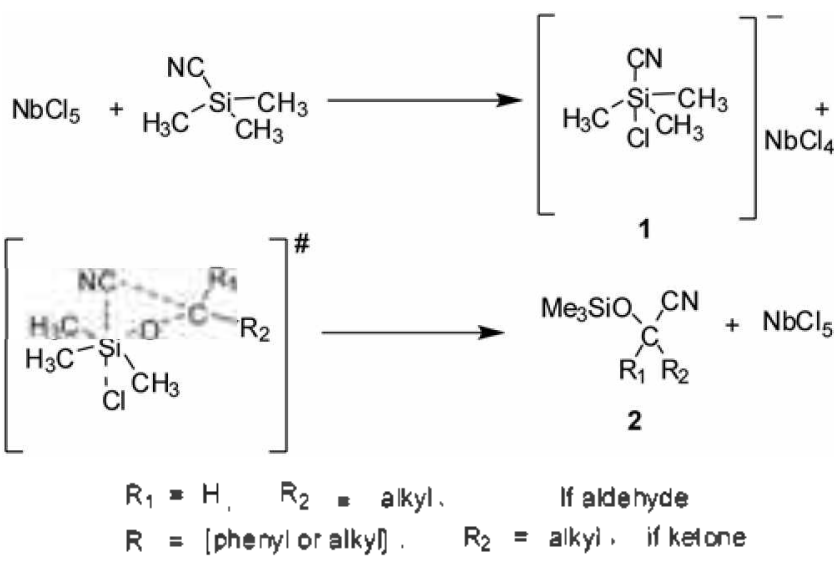

Scheme 1. Mechanism of cyanosilylation of carbonyl compounds catalyzed by $\mathrm{NbCl}_{5}$.

minutes with yield of $97 \%$. The aromatic acetophenone took nearly 46 hours to complete the reaction with yield of $90 \%$. The reactions with the aldelyydes may be facile than with ketones due to the steric reason. The mechanism of cyanosilylation of carbonyl compounds catalyzed by $\mathrm{NbCl}_{5}$ is proposed as follows. $\mathrm{NbCl}_{5}$ can acts as a source of nucleophiles $\mathrm{Cl}^{-}$. When $\mathrm{NbCl}_{5}$ acts a nucelophilic chloride ion. a pentavalent silicon compound is formed (1). which is the reactive species. The hypervalent silicon compound reacts with carbonyl compound so as to give rise to the cyanosily lether (2) (Scheme 1). There are various reports available regarding the fomation of hypervalent silicate ions due to the presence of nuclephiles. ${ }^{8}$ The ${ }^{1} \mathrm{H}$ NMR and ${ }^{13} \mathrm{C}$ NMR spectrum of both TMSCN and a mixture of TMSCN and $\mathrm{NbCl}_{5}$ are monitored. The $\mathrm{CH}_{3}$ peak of TMSCN observed at $\delta 0.354 \mathrm{ppm}$ was found to be shifted to $0.185 \mathrm{ppm}$ in the ${ }^{1} \mathrm{H}$ NMR spectrum of mixture of TMSCN and $\mathrm{NbCl}_{5}$. The ${ }^{13} \mathrm{C}$ spectrum of TMSCN is also shifted from $1.98 \mathrm{ppm}$ to $\delta 2.90$ ppm. The shift of TMSCN peak in both ${ }^{1} \mathrm{H}$ NMR and ${ }^{13} \mathrm{C}$ NMR spectra may be due to the formation of pentavalent silicon compound as suggested in mechanism (Scheme 1).

In conclusion, we have developed a new. mild and efficient cataly'st for cyanosilylation of various carbonyl compounds. The reported procedure clearly demonstrated that $\mathrm{NbCl}_{\xi}$ is an excellent catalyst for the preparation of racemic silylethers in relatively short reaction time with low catalyst loading under solvent-free conditions. The important features of our method are: mild reaction conditions. simple work up. solvent-free condition. inexpensive and readily available cataly'st. The studies are in progress to confinm the mechanistic pathway as well as the reusability of the catalyst $\mathrm{NbCl}_{\text {s. }}$.

\section{Experimental Section}

Silylcyanation of benzaldehyde: 2-phenyl-2-(trimethylsilyloxy)acetonitrle (Table 2. entry 1) A mixture of benzaldehyde (lmmole), dispersed $\mathrm{NbCl}_{5}$ ( $1 \mathrm{~mol} \%$ ) and TMSCN (1.2 equiv.) were stirred for $15 \mathrm{~min}$ at it in a $10 \mathrm{~mL}$ round bottom flask. Then $0.5 \mathrm{~mL}$ of $\mathrm{CH}_{2} \mathrm{Cl}_{2}$ was added to the mixture and stirred for $10 \mathrm{~min}$. The reaction mixture was 
purified by silica gel flash chromatograply by using EtOAchexane (1:9) mixture as eluent. The desired 2-phenyl-2(trimethy lsily loxy)acetonitrile was obtained as colourless oil (yield $99 \%$ ). The yield determined by ${ }^{~} \mathrm{H}$ NMR was $100 \%$. ${ }^{1} \mathrm{H} \mathrm{NMR}\left(\mathrm{CDCl}_{3}, 200 \mathrm{MHz}\right) ; \delta=0.257(\mathrm{~s}, 9 \mathrm{H}) .5 .52(\mathrm{~s}, \mathrm{lH})$. 7.42-7.47 (m. $5 \mathrm{H}) .{ }^{13} \mathrm{C} \mathrm{NMR}(\mathrm{CDCl} 3.100 \mathrm{MHz}): \delta=-0.32$. 63.59. 119.12. 126.29. 128.87, 129.27, 136.18. HRMS (EI): $\mathrm{m} / \mathrm{z}$ calcd. for $\mathrm{C}_{11} \mathrm{H}_{1} \mathrm{NOSi}\left(\mathrm{M}^{-}\right)$: 205.0923 : found: 205.0912 . The other substrates mentioned in Table 2 were also silylcyanted by using the same procedure.

2-(4-Methylphenyl)-2-trimethylsilyloxyacetonitrile (entry 2). ${ }^{1} \mathrm{H} \mathrm{NMR}\left(\mathrm{CDCl}_{3}, 200 \mathrm{MHz}\right.$ ): $\delta=0.142$ (s. $\left.9 \mathrm{H}\right), 2.29$ (s. $3 \mathrm{H}), 5.49(\mathrm{~s}, 1 \mathrm{H}), 7.18(\mathrm{~d}, 2 \mathrm{H}) 7.25(\mathrm{~d}, 2 \mathrm{H}){ }^{13} \mathrm{C}$ NMR $\left(\mathrm{CDCl}_{3}, 100 \mathrm{MHz}\right): \delta=-0.28 .55 .78 .63 .87 .114 .66,119.47$. $127.58,128.78,160.23$. HRMS $(E I): \mathrm{n} / \mathrm{z}$ calcd for $\mathrm{C}_{12} \mathrm{H}_{17} \mathrm{NOSi}\left(\mathrm{M}^{+}\right): 219.1079$ : found: 219.1069 .

2-(3-Methylphenyl)-2(trimethylsilyloxy)acetonitrile (entry 3). ${ }^{\mathrm{H}} \mathrm{H}$ NMR $\left(\mathrm{CDCl}_{3}, 200 \mathrm{MHz}\right): \delta=0.232$ (s. $9 \mathrm{H}$ ), 5.45 (s. $\mathrm{lH}), 2.38-2.42(\mathrm{~m}, 3 \mathrm{H}) 7.26-7.28(\mathrm{~m}, 4 \mathrm{H}){ }^{13} \mathrm{C} \mathrm{NMR}\left(\mathrm{CDCl}_{3}\right.$. $100 \mathrm{MHz}): \delta=0.123,21.46 .63 .72 .119 .21 .123 .41 .126 .93$. 128.74. 130.02, 136.07. 138.74

2-(t-Methoxyphenyl)-2-(trimethylsilyloxy)acetonitrile (entry 4). ${ }^{1} \mathrm{H}$ NMR $\left(\mathrm{CDCl}_{3}, 200 \mathrm{MHz}\right): \delta=0.38$ (s, 9H). $3.83(\mathrm{~s}, 3 \mathrm{H}), 5.44$ (s. $\mathrm{lH}$ ). 6.96 (d. $2 \mathrm{H}) .7 .42$ (d. $2 \mathrm{H}){ }^{13} \mathrm{C}$ NMR $\left(\mathrm{CDCl}_{3} .100 \mathrm{MHz}\right): \delta=-0.26 .55 .34,63.34 .114 .25$. 119.32, 127.93. 128.46. 160.33. HRMS (EI): $\mathrm{m} / \mathrm{z}$ calcd. for $\mathrm{C}_{12} \mathrm{H}_{17} \mathrm{NO}_{-} \mathrm{Si}\left(\mathrm{M}^{-}\right): 235.1029$ : found : 235.1032 .

2-(+-tert-Butylphenyl)-2-trimethylsilyloxy)acetonitrile entry 5). ${ }^{1} \mathrm{H} \mathrm{NMR}\left(\mathrm{CDCl}_{3}, 200 \mathrm{MHz}\right): \delta=0.23$ (s. 9H). 1.32 (s. $9 \mathrm{H}) .5 .38$ (s. $\mathrm{HH}), 7.09-7.21(\mathrm{~m}, 5 \mathrm{H}) .{ }^{13} \mathrm{C} \mathrm{NMR}\left(\mathrm{CDCl}_{3}\right.$. $100 \mathrm{MHz}): \delta=-0.39 .31 .12,34.52 .63 .33 .119 .28 .125 .73$. 126.04. 133.19, 152.47. HRMS (EI): $\mathrm{m} / \mathrm{z}$ calcd for $\mathrm{C}_{15} \mathrm{H}_{3} \mathrm{NOSi}\left(\mathrm{M}^{+}\right): 261.1549$ : found: 261.1552 .

2-(2-Chlorophenyl-)-2-(timethylsilyloxy)acetonitrile (entry 6). ${ }^{1} \mathrm{H} \mathrm{NMR}\left(\mathrm{CDCl}_{3} .200 \mathrm{MHz}\right): \delta=0.252$ (s, 9H). $5.8 \mathrm{l}$ (s. $1 \mathrm{H}) 7.32-7.4$ (m. 3H), $7.72(\mathrm{~d}, \mathrm{IH}){ }^{13} \mathrm{C} \mathrm{NMR}\left(\mathrm{CDCl}_{3}\right.$. $100 \mathrm{MHz}): \delta=-0.206 .60 .75 .127 .46 .128 .26,129.64 .130 .51$.

2-(3-Chlorophenyl-)-2-(timethylsilyloxy)acetonitrile (entry 7). ${ }^{1} \mathrm{H} \mathrm{NMR}\left(\mathrm{CDCl}_{3} .200 \mathrm{MHz}\right.$ ): $\dot{\delta}=0.25$ (s, 9H) 5.42 (s. $1 \mathrm{H}) 7.35-7.37$ (m. 3H). 7.47 (s. $1 \mathrm{H}){ }^{13} \mathrm{C}$ NMR $\left(\mathrm{CDCl}_{3}\right.$. $100 \mathrm{MHz}): \delta=-0.176 .62 .93 .124 .256 .126 .39,129.45,130.14$.

2-(4-Chlorophenyl-)-2-(timethylsilyloxy)acetonitrile (entry 8). ${ }^{1} \mathrm{H}$ NMR ( $\left.\mathrm{CDCl}_{3}, 200 \mathrm{MHz}\right): \delta=0.25$ (s, 9H). $5.48(\mathrm{~s}, \mathrm{lH}) 7.38-7.42(\mathrm{ml}, 4 \mathrm{H}){ }^{13} \mathrm{C}$ NMR $\left(\mathrm{CDCl}_{\hat{3},}, 100 \mathrm{MHz}\right)$ : $\delta=0.161 .63 .01 .118 .71,127.05 .127 .59 .129 .09,134.74$.

HRMS (EI): $\mathrm{m} / \mathrm{z}$ calcd. for $\mathrm{C}_{11} \mathrm{H}_{1+} \mathrm{CINOSi}(\mathrm{M}+$ ): 239.0533 ; found: 239.0539 .

2-(3-Phenoxyphenyl)-2-(trimethylsilyloxy)acetonitrile (entry 9). ${ }^{1} \mathrm{H}$ NMR $\left(\mathrm{CDCl}_{3} .200 \mathrm{MHz}\right): \delta=0.218$ (s, 9H). $5.42(\mathrm{~s}, \mathrm{lH}) .7 .01-7.20(\mathrm{~m}, 5 \mathrm{H}), 7.34-7.38(\mathrm{~m} .4 \mathrm{H}) .{ }^{13} \mathrm{C}$ NMR $\left(\mathrm{CDCl}_{\hat{j}} .100 \mathrm{MHz}\right): \delta=-0.16,63.28 .116 .37 .118 .85$. $119.17,119.30 .120 .64,123.75,129.81 .130 .22,138.08$. 156.39. 157.88 .

2-Furanyl (trimethylsilyloxy)acetonitrile (entry 10), ${ }^{1} \mathrm{H}$ NMR $\left(\mathrm{CDCl}_{3}, 200 \mathrm{MHz}\right): \delta=0.2 \mathrm{l}$ (s. $\left.9 \mathrm{H}\right), 5.58(\mathrm{~s}, \mathrm{lH})$. $6.41-6.43(\mathrm{~m}, 1 \mathrm{H}), 6.57-6.6(\mathrm{~m} . \mathrm{HH}), 7.4-7.52(\mathrm{~m} . \mathrm{lH}){ }^{13} \mathrm{C}$ NMR $\left(\mathrm{CDCl}_{3}, 100 \mathrm{MHz}\right) ;-0.42 .57 .42 .109 .71,110.76$.
117.12. 143.87, 148.23. HRMS (EI): $\mathrm{m} / \mathrm{z}$ calcd. for $\mathrm{C}_{9} \mathrm{H}_{13} \mathrm{NO}_{2} \mathrm{Si}\left(\mathrm{M}^{+}\right)$: 195.0715 ; found: 195.0712 .

2-(Naphthalene-1-yl)-2-(trimethylsilyloxy)acetonitrile (entry 11). ${ }^{1} \mathrm{H}$ NMR $\left(200 \mathrm{MHz} . \mathrm{CDCl}_{3}\right): \delta=0.226$ (s. $9 \mathrm{H}$ ), 6.05 (s. $\mathrm{lH}$ ). 7.45-7.7 (m.3H) 7.85-7.95 (m. 3H). 8.23 (d. 1H) ${ }^{13} \mathrm{C} \mathrm{NMR}\left(\mathrm{CDCl}_{3} .100 \mathrm{MHz}\right): \delta=-0.285 .63 .4 .118 .45$, 122.37, 125.62. 125.01, 126.3. 128.3, 131.01. 133.45, 136.12.

3-Methyl-2-trimethylsilyloxybutanenitrile (entry 12). ${ }^{1} \mathrm{H}$ NMR (200 MHz. CDCl $): \delta=0.2(\mathrm{~s}, 9 \mathrm{H}) .0 .88-1.05(\mathrm{~m}$, $6 \mathrm{H}) .1 .94-1.96(\mathrm{~m}, \mathrm{lH}) .4 .16(\mathrm{~d} . \mathrm{lH}){ }^{13} \mathrm{C}$ NMR $\left(\mathrm{CDCl}_{3}, 100\right.$ $\mathrm{MHz}): \delta=-0.335,17.68,33.921 .67 .28,119.94$.

2-(Trimethylsilyloxy) pent-3-enenitrile) (entry 13). ${ }^{1} \mathrm{H}$ NMR (CDCl. $200 \mathrm{MHz}$ ): $\delta=0.24$ (s. $9 \mathrm{H}$ ). 1.74 (d. $3 \mathrm{H}$ ). 4.90 (d. $\mathrm{lH}), 5.51-5.62(\mathrm{~m}, \mathrm{lH}), 5.93-6.04(\mathrm{~m} . \mathrm{lH}) .{ }^{13} \mathrm{C}$ NMR $\left(\mathrm{CDCl}_{3} .100 \mathrm{MHz}\right): \delta=-0.40 .17 .17,61.92,118.45 .126 .06$. 130.88. HRMS (EI): $\mathrm{m} / \mathrm{z}$ calcd. for $\mathrm{C}_{\$} \mathrm{H}_{15} \mathrm{NOSi}\left(\mathrm{M}^{-}\right)$: 169.0922: found: 169.0917 .

(E)-4-Phenyl-2-(trimethylsilyloxy)but-3-enenitile (entry 14). ${ }^{1} \mathrm{H}$ NMR (CDCl. $200 \mathrm{MHz}$ ) $\delta=0.32$ (s. 9H). 5.18-5.19 (d. lH), 6.18-6.23 (m. lH), 6.78-6.81 (d, lH) 7.28-7.42 (m. $5 \mathrm{H}){ }^{13} \mathrm{C} \mathrm{NMR}\left(\mathrm{CDCl}_{3} .100 \mathrm{MHz}\right): \delta=-0.26,64.2 .118 .3$, 127.5, 127.8. 128.3, 128.4, 128.6, 136.2. HRMS (EI): $\mathrm{m} / \mathrm{z}$ calcd for $\mathrm{C}_{13} \mathrm{H}_{17} \mathrm{NOSi}\left(\mathrm{M}^{+}\right)$: 231.1079 ; found: 231.1082 .

1-(Trimethylsilyloxy)-2-cyclohexenecarbonitrile (entry 15). ${ }^{1} \mathrm{H}$ NMR ( $\left.\mathrm{CDCl}_{3}, 200 \mathrm{MHz}\right): \delta=0.24(\mathrm{~s}, 9 \mathrm{H}), 1.74-1.86$ (m, 2H), 1.91-1.98 (m. 2H), 2.04-2.18 (m, 2H) $5.72 .5 .8(\mathrm{~m}$. lH) $5.97 .5 .99(\mathrm{~d}, \mathrm{lH}) .{ }^{12} \mathrm{C}$ NMR $\left(\mathrm{CDCl}_{3} .100 \mathrm{MHz}\right): \delta=$ $1.62,18.45,24.39 .37 .00,66.50,121.80,127.5$. and 132.5 HRMS (EI): $\mathrm{m} / \mathrm{z}$ calcd for $\mathrm{C}_{10} \mathrm{H}_{17} \mathrm{NOSi}\left(\mathrm{M}^{-}\right)$: 195.1079 : found: 195.1072 .

2-(Trimethylsilyloxy)-2-methyloctanenitrile (entry 16). ${ }^{1} \mathrm{H}$ NMR (CDCl $3.200 \mathrm{MHz}$ ): $\delta=0.22$ (s. 9H), 0.87-0.91 (t, $3 \mathrm{H}) 1.29-1.32(\mathrm{~m} .8 \mathrm{H}), 1.56(\mathrm{~s}, 3 \mathrm{H}) 1.68-1.7 \mathrm{i}(\mathrm{m} .2 \mathrm{H}){ }^{13} \mathrm{C}$ NMR (CDCl, $100 \mathrm{MHz}): \delta=1.42 .14 .12 .22 .62,24.32$, 28.98. 29.05, 31.69. 43.44. 69.70, and 122.16. HRMS (EI): $\mathrm{m} / \mathrm{z}$ calcd for $\mathrm{C}_{12} \mathrm{H}_{2} \mathrm{NOSi}(\mathrm{M}+$ ): 227.1705 : found: 227.1710 .

2-Trimethylsilyloxy-2-phenylpropanenitrile (entry 17). ${ }^{1} \mathrm{H} \mathrm{NMR} \mathrm{CDCl}_{3} .200 \mathrm{MHz}$ ): $\delta=0.16$ (s, 9H), 1.84 (s. $\left.3 \mathrm{H}\right)$, $7.36-7.55(\mathrm{~m}, 5 \mathrm{H}){ }^{13} \mathrm{C}$ NMR $\left(\mathrm{CDCl}_{3}, 100 \mathrm{MHz}\right): \delta=0.89$. 33.42. 71.46, 121.45, 124.46, 128.48, 141.87 HRMS (EI): $\mathrm{m} / \mathrm{z}$ calcd for $\mathrm{C}_{1} \mathrm{H}_{17} \mathrm{NOSi}\left(\mathrm{M}^{+}\right)$: 219.1079: found: 219.1072 .

Trimethyl silamecarbonitrile (TMSCN). ${ }^{\mathrm{H}} \mathrm{NMR}\left(\mathrm{CDCl}_{3}\right.$, $200 \mathrm{MHz}$ ): $\delta=0.354$.

${ }^{13} \mathrm{C} \mathrm{NMR}\left(\mathrm{CDCl}_{3} .100 \mathrm{MHz}\right): \delta=1.98 .126 .97$.

A mixture of Trimethyl silanecarbonitrile and $\mathrm{NbCl}_{\mathbf{5}}$. ${ }^{1} \mathrm{H}$ NMR $\left(\mathrm{CDCl}_{3,2} 200 \mathrm{MHz}\right) ; \delta=0.185$.

${ }^{13} \mathrm{C} \mathrm{NMR}\left(\mathrm{CDCl}_{3} .100 \mathrm{MHz}\right): \delta=2.90 .127 .23$.

Acknowledgments. We warmly thank the Biological modulators for the financial support. Korea Research foundation should be mentioned for BK2l povided to Inha University

\section{References}

1. (a) Gregon; R. J. H. Chem. Rev 1999, 99. 3649. (b)Special symposium for the synthesis of nonracemic eyanohydrins, see: Tetrahedron 2004, 60, 10371. (c) Brunel. J. M.; Holmes. I. P. 
Angew Chem. Int Ed. 2004. 43. 2752. (d) Kanai. M.: Kato. N.: Ichikawa. E.: Shibasaki. M. Stztet 2005. 1491.

2. (a) Bandini. M.: Cozzi. P. G.: Garelli. A.: Melchiorre. P.: UmaniRonchi, A. Eur. J. Org. (Them. 2002, 3243. (b) Cordoba, R.: Plumet, J. Tehahedron Lett. 2003. H4, 6157. (c) Chen. F.-X.: Liu, X.: Qin. B.: Zhoul. H.: Feng. X.: Zhang. G. Srmthesis 2004, 14, 2266. (d) Aspinall. H. C.: Bickley. J. F.: Greeves. N.: Kelly. R. V: Smith. P. M. Organonetallics 2005. 24. 3458. (e) Liu. Y.: Liu. X: Xin. J.: Feng. X. Sinlen 2006. 1085.

3. (a) Hamashina, Y: Sawada. D.: Kanai. M: Shibasaki. M. J. Am. Chem. Soc, 1999. 121, 2641. (b) Hamashima, Y: Kanai, M: Shibasaki. M. J. Am. Chem. Soc. 2000. 122, 7412 (c) Chen, F.: Feng. X.: Oin. B.: Zhang. G. Jiang. Y. Organic Lett. 2003. 5. 949. (d) Ryu. D. H.: Corey. E. J. J.Am. Chem. Soc. 2005. 127.5384. (e) Fuerst. D. E.: Jacobsen. E. N. J. Am. Chem. Soc. 2005. 127. 8964. (f) Hatano. M.: Ikeno. T: Miyanoto. T: Ishihara. K. J. Am. Chem. Soc. 2005. 127, 10776. (g) Harada. T.: Kanda. K. Organic lett. $2006,8,3817$.

4. (a) Kim. S. S.: Song. D. H. Eur, J. Org Chem. 2005. 1777. (b) Kim. S. S.: Lee. S. H. Synth. Conmm 2005 35.751. (c) Kim. S. S.: Lee. S. H.: Kwak. J. M. Tetrohedron: Astmmetrn 2006. 17, 1165. (d) Kim, S. S. Pure Appl. Chem. 2006, 78, 977. (e) Kim. S. S. Kwak. I. M. Tetratredron 2006. 62, 49. (f) Kim, S. S.: Kwak, J. M.: Rajagopal, G. Bull. Koream (them. Soc. 2006, 27. 1638 .

5. (a) Kim. S. S.: Rajagopal. G.: Song. D. H. J. Organonet. Chem. 2004. 689. 1734. (b) Kim. S. S.: Kim. D. W: Rajagopal. G. Symhesis-Stuttgart 2004, 213 . (c) Kim, S. S.: Rajagopal, G.: Kim. D. W. Song. D. H. Symth. Commm. 2004, 34, 2973. (d) Rajagopal. G.: Kim. S. S. Kwak. J. M. Bull. Norean (Chem. Soc.
2006. 27. 1907. (e) Kim. S. S.: Rajagopal. G. Swnthesis-stuftgart 2007. 2. 215. (f) Kim. S. S.: Rajagopal. G: George. S. C. Appl. Organontet. Chent 2007. 21. 198.

6. (a) Lidy. W: Sundermever, W. Chem. Ber: 1973, 106, 587, (b) Evans. D. A.; Truesdale. L. K. Tetrahedron Lett 1973, 4929. (c) Komatsu. N.: UJda. M.: Suzuki. H.: Takahashi. T.: Domae. T: Wada. M. Tetrohedron Lett. 1997. 38. 7215. (d) Reetz. M. T.: Drewes. M. W. Harms. K: Reif. W. Tetrdhedron Leth. 1988. 29. 3295. (e) Loh. T.-P: Xu. K.-C.: Ho. D. S. C.: Sim. K.-Y. Swlett 1998. 369. (f) Vougioukas. A. E.: Kagan. H. B. Tetrahedron Lett. 1987. 28,5513 . (g) Evans, D. A.; Truesdale, L. K.: Carroll. G. L. J. Chem. Soc. Chem. Commu 1973. 55. (h) Iwanami, K.: Aovagi. M.: Oriyama. T. Tetrahedron Lett. 2005. 46. 7487, (i) Kurono. N.: Yamaguchi. M.: Suzuki. K.: Ohkuma. T. J. Org. Chent 2005. 70. 6530 .

7. (a) Howarth, J:- Gillespie. K. Tetrahedron Lett. 1996.37,6011. (b) Andrade, C. K. Z.: Oliveira. G. R. Tetrahedron Lett. 2002. 43. 1935. (c) Andrade. C. K. Z.: Azevedo, N. R. Tetrahedron Lett. 2001. 42. 6473. (d) Guo. Q: Mivaji. T.: Hara. R.: Shen. B.: Takahashi. T. Tetrahedron 2002. 58.7327. (e) Ortiz. A.: Quintero. L.: Hernandez. H.: Maldonado. S.: Mendoza. G.: Bernes. S. Tetrahedron Lett. 2003, 4f, 1129. (f) Arai. S.; Sudo, Y.: Nishida. A. Synlett 2004, 6. 1104. (g) Yadav. J. S.: Subba Reddy. B. V: Eeshwaraiah, B.; Reddy. P. N. Tetrahedron 2005. 61, 875. (h) Yadav. J. S.: Narsaiah. A. V: Subba Reddy. B. V.: Basak. A. K. Nagaiah. K. J. Mol. Cat A: Chemi. 2005. 230. 107.

8. (a) Liu. X: Oin. B.: Zhou. X: He. B.: Fenn. X. J. Am. Chem. Soc. 2005. 127. 12224. (b) Kabavashi. S.: Nishio. K. J. Org. Chem 1994. 59,6620 (c) Chuit, C.; Corriu, R. J. P: Reve, C: Young, J. C. Chem Rev 1993.93,1371. 